\title{
Metastatic prostate cancer cells in lymph nodes perturb nodal laminin expression
}

\author{
M. Shkurnikov*, D. Maltseva \\ National Medical Research Radiology Center, Obninsk, Russia \\ *e-mail:mshkurnikov@imscs.msu.ru
}

Key words: prostate cancer, metastasis, microarrays, laminins

Motivation and Aim: Metastasis of the most epithelial tumors occurs primarily through the lymphatic system. It is supposed that the presence of tumor cells in the lymph nodes (LNs) is not only a marker, but also an important mediator of metastatic disease. Normal stromal infrastructure of LN is a crucial for orchestrating immune cell homeostasis and adaptive immunity. Extracellular matrix (ECM) proteins play the most important role in LN parenchyma organization. Here, we compared the expression profile of genes encoding ECM components in LNs with and without histologically confirmed metastatic cells from a patient with prostate cancer.

Methods and Algorithms: Six LNs with and without histologically confirmed metastatic cells were obtained surgically from a patient with prostate cancer. A comparative transcriptomic analysis was performed using the microarray technology. The differential expression of the most relevant ECM protein genes was confirmed by qPCR.

Results: Decreased expression levels of genes encoding laminin 411 (LM-411) chains (LAMA4 - 3.4 times, LAMB1 - 2.3 times, LAMC1 - 2.2 times) was revealed in LNs with metastatic cells. Lymphocytes penetrate the blood capillary wall only at that places where the basement membrane (BM) contains LM-411. Thus, the reduction of LM-411 expression may disrupt the transport of naïve lymphocytes in LNs. LM-411 is also one of the two laminins comprising the BM of the conduit system, a special system that provides closer contact of LN resident dendritic cells (DCs) with lymph and antigens therein. The reduction of LM-411 expression may prevent contact of DCs with antigens, lymphocyte migration through LN space to find the antigen-bearing DCs and further activation and maturation of naïve T cells. A reduced mRNA level of other important BM component collagen type IV (1.7 and 1.6-fold lower expression of $\alpha 1$ and $\alpha 2$ subunits, respectively) was also shown in LNs with metastatic cells. Such decrease of the ECM protein expressions is apparently compensated by upregulation of the others, e.g. $\alpha 2$ laminin chain (1.6 times), fibronectin (2.5 times), collagen type I $\alpha 1$ and $\alpha 2$ subunits (1.4 times), collagen type VI $\alpha 3$ subunit (1.6 times), collagen type XIV $\alpha 1$ subunit (1.7 times). An increased collagen expression was earlier detected in LNs with metastatic cells in breast cancer.

Conclusion: The presence of metastatic cells in LNs may perturb their normal infrastructure and function. This might be one of the mechanisms used by tumor to suppress immune system.

Acknowledgements: Supported by the MES of Russia (14.616.21.0092, RFMEFI61618X0092). 